\title{
Fatores de correção para perímetro escrotal ao sobreano para tourinhos mestiços Aberdeen Angus x Nelore
}

[Adjustment factors for scrotal circumference at yearling for crossbred Aberdeen Angus x Nelore young bulls]

\author{
J.S. Lopes ${ }^{1}$, P.R.N. Rorato ${ }^{2 *}$, T. Weber ${ }^{1}$, J.G. Comin $^{3}$, R.O. Araújo ${ }^{1}$ \\ ${ }^{1}$ Aluno de pós-graduação - UFSM - Bolsista CAPES \\ ${ }^{2}$ Departamento de Zootecnia - UFSM \\ Av. Roraima, 1000 \\ 97105-900 - Santa Maria, RS \\ ${ }^{3}$ Aluna de graduação - UFSM - Bolsista PIBIC
}

\begin{abstract}
RESUMO
Obtiveram-se fatores de correção (FC) para o perímetro escrotal ao sobreano (PES) para os efeitos de grupo genético (GG), heterozigose individual (HI), peso ao sobreano (PS) e idade do animal à pesagem de sobreano (IDS), utilizando-se registros de peso corporal e medidas de perímetro escrotal obtidos de 11.662 tourinhos das raças Aberdeen Angus, Nelore e de produtos do cruzamento entre elas, criados nas regiões Sul, Sudeste e Centro-Oeste do Brasil, nascidos entre 1987 e 2001. Os coeficientes de regressão que geraram os FC foram estimados pelo método dos quadrados mínimos, adotando um modelo que incluiu os efeitos de grupo de contemporâneos ao sobreano (GC), GG, heterozigose materna (HM), HI, PS e IDS. Todos os efeitos incluídos no modelo foram significativos $(\mathrm{P}<0,01)$ sobre PES. A média observada e respectivo desvio-padrão para PES foi $29,90 \pm 3,55 \mathrm{~cm}$. Foi observado efeito polinomial de segunda ordem de PS sobre PES. Foram verificados ganhos decrescentes no PES à medida que o POS aumentou, ou seja, os maiores acréscimos foram observados em animais recém desmamados. O efeito linear de PS sobre PES foi $0,06695804 \pm 0,00345000 \mathrm{~cm} / \mathrm{kg}$, e o efeito quadrático, $0,00005252 \pm 0,00000508 \mathrm{~cm} / \mathrm{kg}^{2}$. Para IDS o efeito linear foi de $0,02176450 \pm 0,00038568 \mathrm{~cm} / \mathrm{dia}$. Aumentos de HI e HM promoveram incremento no PES e de GG, decréscimos. Os fatores incluídos no modelo foram importantes fontes de variação que devem ser considerados no ajuste de PES para seleção de tourinhos para melhora da precocidade sexual.
\end{abstract}

Palavras-chave: bovino de corte, coeficiente de regressão, heterozigose, população multirracial, precocidade sexual

\begin{abstract}
Adjustment factors (AF) for scrotal circumference at yearling (SCY) were figured out for effects of genetic group $(G G)$, individual heterozygosis $(I H)$, yearling weight $(Y W)$, and age of the animal at yearling weight $(A Y W)$ using body weight and scrotal circumference records from 11,662 Aberdeen Angus, Nelore, and their crosses. The animals were born from 1987 to 2001 and were raised in the South East and Central West Regions of Brazil. The regression coefficients to obtain AF were estimated by least squares means method. The model included the fixed effects of contemporaneous group at yearling (CG), maternal heterozygosis (MH), IH, and the covariates $Y W$ (linear and quadratic effects) and $A Y W$ (linear effect). All the factors included in the model showed significant effects $(P<0.01)$ on SCY. The mean and standard deviation for SCY were $29.90 \pm 3.55 \mathrm{~cm}$. Quadratic effect of $Y W$ on $S C Y$ was also observed. Decreases in SCY with the increase in $Y W$ was found. High SCY was observed immediately after post-weaning. The YW effects on SCY were $0.06695804 \pm 0.00345000 \mathrm{~cm} / \mathrm{kg}$ (linear effect) and $-0.00005252 \pm 0.00000508 \mathrm{~cm} / \mathrm{kg}^{2}$ (quadratic effect). The AYW linear effect on SCY was $0.02176450 \pm 0.00038568 \mathrm{~cm} /$ day. The factors included in the model are important sources of variation to adjust SCY for the selection of young bulls in order to improve sexual precocity.
\end{abstract}

Keywords: beef cattle, regression coefficient, heterozygosis, multibreed population, sexual precocity

Recebido em 27 de maio de 2008

Aceito em 4 de fevereiro de 2009

* Autor para correspondência (corresponding author)

E-mail: rorato@smail.ufsm.br 


\section{INTRODUÇÃO}

A cadeia produtiva da carne bovina vem se tornando mais competitiva a cada ano, com consequente redução na margem de lucro; isto tem levado pecuaristas e pesquisadores a buscarem alternativas que aumentem a produtividade do sistema. Estudos têm sido realizados no sentido de aumentar a velocidade, tanto de crescimento como de maturidade sexual. Nesse sentido, vem sendo utilizado o cruzamento entre raças, buscando aproveitar os benefícios da heterose, da combinação aditiva e da complementaridade entre as raças para melhorar a eficiência da produção e a qualidade do produto.

Considerando que a adoção de estações de monta dificulta a verificação da precocidade sexual das fêmeas (Ortiz Peña et al., 2000), aliada às baixas intensidades de seleção normalmente impostas a elas, espera-se um pequeno progresso genético para as características reprodutivas (Alencar et al., 1993), sendo importante promover estudos relacionados à função reprodutiva dos machos.

$\mathrm{Na}$ busca de alternativas para aumentar a precocidade sexual em bovinos, os programas de melhoramento genético vêm incluindo, como critério de seleção, a medida do perímetro escrotal. Segundo Brito (1997), a utilização da medida do perímetro escrotal em análises genéticas tem como vantagens a facilidade de mensuração, a confiabilidade nas medidas e a alta herdabilidade, além de estar correlacionada com as características de crescimento e reprodução, em ambos os sexos.

A circunferência escrotal, quando mensurada em animais jovens, está associada ao tamanho testicular (Coulter e Keller, 1982), à capacidade de produção espermática, às características físicas do sêmen, à idade à puberdade e, também, à fertilidade dos machos (Alencar et al., 1993), bem como à das fêmeas aparentadas, principalmente meias irmãs e filhas (Toelle e Robinson, 1985). Teixeira et al. (1998) afirmaram ser necessário que os programas de melhoramento genético ofereçam ao produtor tanto as medidas de perímetro escrotal ajustadas para peso corporal e idade, como as ajustadas apenas para idade, pois podem existir diferentes prioridades em relação à precocidade de crescimento e precocidade sexual, ainda que essas duas características estejam correlacionadas entre si. Dal-Farra et al. (2000) definiram um modelo para ajuste de medidas de perímetro escrotal para animais de diferentes grupos genéticos das raças Angus e Nelore. Os autores consideraram, além dos efeitos da idade e do peso corporal do animal, os efeitos de grupo de contemporâneos ao sobreano e da heterozigose individual.

O objetivo deste trabalho foi estimar fatores de correção para medida do perímetro escrotal ao sobreano para grupo genético, heterozigose individual, peso ao sobreano e idade do animal à pesagem de sobreano em rebanhos formados pelas raças Aberdeen Angus, Nelore e de seus cruzamentos.

\section{MATERIAL E MÉTODOS}

Foram utilizados registros de peso e de perímetro escrotal de 11.662 tourinhos das raças Aberdeen Angus, Nelore e de produtos do cruzamento entre elas, filhos de 605 touros e de 7.312 vacas, nascidos em 37 fazendas, nas regiões Sul, Sudeste e Centro-Oeste do Brasil, entre 1987 e 2001. O banco de dados foi fornecido por Gensys Consultores Associados S/C Ltda. e Natura Genética Sul-Americana.

$\mathrm{Na}$ montagem do arquivo de trabalho, foram geradas as variáveis: 1 - estação de nascimento (EN), num total de quatro - EN1, reunindo os animais nascidos nos meses de janeiro a março, EN2, os nascidos de abril a junho, EN3, os nascidos de julho a setembro e EN4, os nascidos de outubro a dezembro -; 2 - peso ao sobreano ajustado para 550 dias de idade (P550); 3 - grupo de contemporâneos (GC), composto pelos animais nascidos na mesma fazenda, ano e estação e pertencentes ao mesmo grupo de manejo ao sobreano, totalizando 200 grupos distintos; 4 - grupo genético (GG), formado pela fração da raça Nelore representada no genótipo do animal, variando de 0 (Aberdeen Angus puro) a 1 (Nelore puro); 5 - heterozigose materna (HM) e 6 - heterozigose individual (HI), as duas últimas calculadas segundo a equação descrita por Dickerson (1973).

A distribuição dos animais quanto ao grupo genético (GG) e à heterozigose individual (HI) é apresentada na Tab. 1 . 
Fatores de correção para perímetro...

Tabela 1. Distribuição dos animais em relação ao grupo genético e à heterozigose individual

\begin{tabular}{cccc}
\hline Grupo genético & $\mathrm{N}^{\circ}$ de animais & Heterozigose individual & $\mathrm{N}^{\circ}$ de animais \\
\hline 0,0000 & 942 & 0,0000 & 955 \\
0,1875 & 67 & 0,2500 & 10 \\
0,2500 & 115 & 0,3750 & 146 \\
0,3125 & 626 & 0,5000 & 237 \\
0,3750 & 4330 & 0,6250 & 1500 \\
0,4375 & 1027 & 0,7500 & 4513 \\
0,5000 & 3217 & 0,8750 & 1084 \\
0,6250 & 183 & 1,0000 & 3217 \\
0,6875 & 874 & - & - \\
0,7500 & 122 & - & - \\
Outros & 159 & - & - \\
\hline
\end{tabular}

Foram excluídos do banco de dados os grupos de contemporâneos com menos de cinco animais, os reprodutores com menos de cinco filhos e com filhos em um só grupo de contemporâneos e os animais com registros incompletos.

As análises estatísticas foram realizadas por meio da metodologia de quadrados mínimos, utilizando o Procedimento GLM, do pacote estatístico SAS/2001.

A significância estatística de cada um dos efeitos foi verificada por uma análise de variância sob um modelo que considerou os efeitos do GC, GG, HM e HI e, como covariáveis, o peso ao sobreano (PS) e a idade da vaca ao parto, componentes linear e quadrático, e a idade do animal na pesagem de sobreano (IDS), componente linear.

A idade da mãe apresentou significância estatística, todavia, influiu pouco no coeficiente de determinação, por isso não foi incluída no modelo final de ajuste, como também não teve fatores de correção calculados conforme sugerido por Dal-Farra et al. (1998), fundamentado na afirmativa de Lunstra et al. (1988) de que grande parte do efeito da idade da mãe sobre o perímetro escrotal decorre, fundamentalmente, da influência desta sobre o peso do animal.

O modelo estatístico adotado considerou os fatores $\mathrm{GC}, \mathrm{GG}, \mathrm{HM}$ e $\mathrm{HI}$, e como covariáveis, PS (efeitos linear e quadrático) e IDS (efeito linear), além do efeito residual aleatório. A inclusão de PS no modelo de análise como covariável, segundo Pereira et al. (2001), se justifica, pois os maiores perímetros escrotais (PES) estão relacionados aos animais mais pesados. De acordo com Ortiz-Peña et al. (2000), ao sobreano espera-se que a cada $100 \mathrm{~kg}$ de ganho de peso, o PES cresça, em média, ao redor de $7,08 \mathrm{~cm}$. Para IDS, a cada 100 dias espera-se que o PES cresça, aproximadamente, $3,83 \mathrm{~cm}$. O modelo de regressão adotado é descrito como:

$\mathrm{PES}_{\mathrm{ij}}=\mathrm{b}_{0}+\mathrm{GC}_{\mathrm{i}}+\mathrm{b}_{1}\left(\mathrm{GG}_{\mathrm{ij}}\right)+\mathrm{b}_{2}\left(\mathrm{HM}_{\mathrm{ij}}\right)+\mathrm{b}_{3}\left(\mathrm{HI}_{\mathrm{ij}}\right)$ $+b_{4}\left(P_{i j}\right)+b_{5}\left(P^{2}{ }_{i j}\right)+b_{6}\left(\operatorname{IDS}_{i j}\right)+e_{i j k}$, em que: $\mathrm{PES}_{\mathrm{ij}}$ é o perímetro escrotal do j-ésimo animal pertencente ao i-ésimo GC; $b_{0}$ é o intercepto do modelo; $\mathrm{GC}_{\mathrm{i}}$ é o efeito do i-ésimo GC; GG é o grupo genético do j-ésimo animal pertencente ao i-ésimo GC; HM é a heterozigose materna do jésimo animal pertencente ao i-ésimo GC; HI é a heterozigose individual do j-ésimo animal pertencente ao i-ésimo GC; PS é o peso ao sobreano, efeito linear, do j-ésimo animal pertencente ao i-ésimo GC; $\mathrm{PS}^{2}$ é o peso ao sobreano, efeito quadrático, do j-ésimo animal pertencente ao i-ésimo GC; IDS é a idade à pesagem de sobreano do j-ésimo animal pertencente ao i-ésimo GC; $b_{1}, b_{2}, b_{3}, b_{4}, b_{5}$ e $b_{6}$ são os coeficientes de regressão do modelo; $\mathrm{e}_{\mathrm{ij}}$ é o erro aleatório associado à ij-ésima observação.

Os fatores de correção para PES foram calculados utilizando-se as soluções obtidas para cada efeito, no ajuste dos polinômios, sendo: $\mathrm{FCx}=\mathrm{PES}_{\mathrm{base}} / \mathrm{PES}_{\mathrm{x}}$, em que: $\mathrm{FC}_{\mathrm{x}}=$ fator de 
correção do ponto $\mathrm{X}$ em função da base; $\mathrm{PES}_{\text {base }}$ $=$ perímetro escrotal ao sobreano estimado na base; e $\mathrm{PES}_{\mathrm{x}}=$ perímetro escrotal ao sobreano estimado no ponto $\mathrm{X}$. Os fatores de correção multiplicativos foram calculados utilizando-se como padrão $330 \mathrm{~kg}$ para peso ajustado aos 550 dias de idade, além de HI e GG iguais a zero.

\section{RESULTADOS E DISCUSSÃO}

As médias e os desvios-padrão observados para as PES, POS e IDS foram: $29,90 \pm 3,55 \mathrm{~cm}$, $326,93 \pm 59,21 \mathrm{~kg}$ e $505,37 \pm 70,59$ dias, respectivamente. A média observada para PES neste trabalho foi de 7 e $6 \%$ mais alta que a relatada por Dal-Farra et al. (1998) e por OrtizPeña et al. (2000), respectivamente, para a raça Nelore, e cerca de $3 \%$ maior que a verificada por Dal-Farra et al. (2000), para uma população Angus x Nelore. O valor de PS foi de 9, 16 e $15 \%$ maior, e o da IDS foi de 22,6 e $19 \%$ menor do que os relatados por Dal-Farra et al. (1998, 2000) e Ortiz-Peña et al. (2000), respectivamente.

O resumo da análise de variância está apresentado na Tab. 2. Nela observa-se que todos os fatores incluídos no modelo apresentaram efeitos significativos sobre PES ( $\mathrm{P}<0,01)$. O PS apresentou efeitos linear e quadrático sobre PES, semelhante ao verificado por Brito (1997), para a raça Hereford; por Dal-Farra et al. (1998), para a raça Nelore e por Dal-Farra et al. (2000), para uma população Angus x Nelore. Ortiz-Peña et al. (2000), para a raça Nelore, relataram significância apenas linear. A IDS apresentou efeito linear sobre PES, semelhante aos resultados de Ortiz-Peña et al. (2000).

A equação definida para o ajuste do perímetro escrotal ao sobreano foi: $\mathrm{PES}=5,09707066+\mathrm{GC}$ $-5,06070718(\mathrm{GG})+0,98226200(\mathrm{HM})+$ $1,62704900(\mathrm{HI})+0,06695804(\mathrm{PS}) \quad-$ $0,00005252\left(\mathrm{PS}^{2}\right)+0,02176450($ IDS $)$.

O coeficiente de regressão para HM indica que os filhos de mães mestiças F1 apresentaram $1 \mathrm{~cm}$ a mais de PES do que os filhos de mães de raça pura. Essa função é, provavelmente, da maior habilidade materna das mães mestiças em relação às puras, as quais, segundo Cundiff et al. (1974) e Skrypzeck et al. (2000), tendem a apresentar maior produção de leite e, portanto, propiciam condições mais favoráveis para seus filhos expressarem todo o seu potencial genético.

Tabela 2. Análise de variância referente ao modelo definido para o ajuste do perímetro escrotal ao sobreano

\begin{tabular}{lcccc}
\hline $\begin{array}{l}\text { Fonte de } \\
\text { variação }\end{array}$ & $\mathrm{gl}$ & $\begin{array}{c}\text { Quadrados } \\
\text { médios }\end{array}$ & $\mathrm{b}$ & $\mathrm{R}^{2}$ \\
\hline Modelo & 205 & $415,68^{*}$ & & 0,58 \\
GC & 199 & $95,26^{*}$ & $-0,0017898$ & \\
GG & 1 & $489,54^{*}$ & $-5,0607072$ & \\
HM & 1 & $97,11^{*}$ & 0,9822620 & \\
HI & 1 & $85,04^{*}$ & 1,6270490 & \\
PS & 1 & $2045,86^{*}$ & 0,0669580 & \\
PS & 1 & $697,56^{*}$ & $-0,0000525$ & \\
IDS & 1 & $323,16^{*}$ & 0,0217645 & \\
Resíduo & 11.456 & 5,41 & & \\
Total & 11.661 & & & \\
\hline
\end{tabular}

*(P<0,01). GC: grupo de contemporâneos; GG: grupo genético do animal; HM: heterozigose materna; HI: heterozigose individual; OS: peso ao sobreano; $\mathrm{PS}^{2}$ : $(\mathrm{PS})^{2}$; IDS: idade à pesagem ao sobreano.

Com relação à $\mathrm{HI}$, a diferença predita entre o PES dos machos da geração F1 e a média do PES dos animais puros foi $1,63 \mathrm{~cm}$, que resultou em heterose de 5,4\%. Estes valores foram mais altos que os observados por Dal-Farra et al. (2000), para uma população Angus x Nelore, os quais relataram diferença de $1,18 \mathrm{~cm}$ e $3,9 \%$ de heterose, e ao relatado por Gregory et al. (1995), para diferentes compostos, de $1,3 \mathrm{~cm}$ em média.

Os valores preditos para PES em função das variações no PS e na IDS são apresentados na Fig. 1. 
a)

\section{PES (cm)}

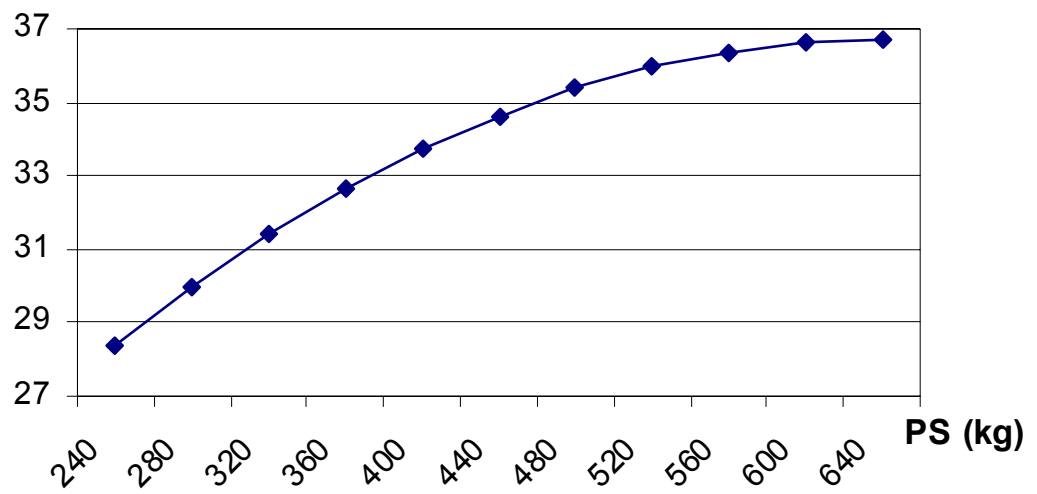

b)

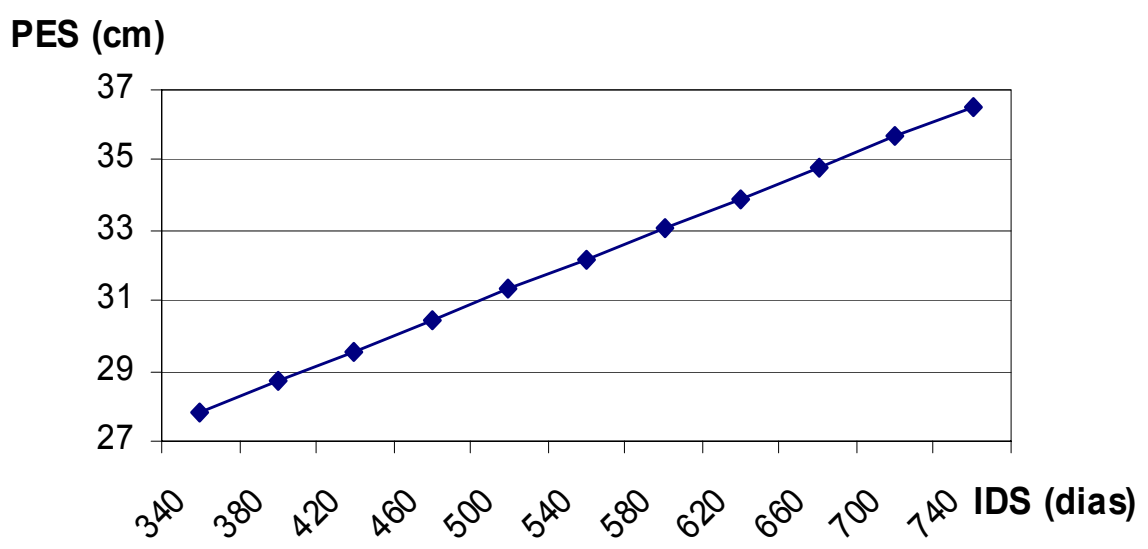

Figura 1. Valores preditos para perímetro escrotal ao sobreano (PES), em função de: a) peso do animal ao sobreano (PS) e b) idade à pesagem de sobreano (IDS).

Foram observados decréscimos no PES à medida que o PS aumentou (Fig. 1a), ou seja, os maiores valores foram preditos para animais recém desmamados e descritos por uma curva sigmoide, semelhante ao encontrado por Bergmann (1999). Os animais atingiram $28 \mathrm{~cm}$ de PES, aproximadamente, aos 360 dias de idade e $240 \mathrm{~kg}$ de PS. Estes valores diferem dos relatados por Dal-Farra et al. (1998) para a raça Nelore, por Dal-Farra et al. (2000), para mestiços das raças Angus x Nelore, e por Ortiz-Peña et al. (2000), para a raça Nelore, no Paraguai. Segundo esses autores, os animais atingiram $28 \mathrm{~cm}$ de PES aos 580, 480 e 350 dias, respectivamente. Embora a idade relatada por Ortiz-Peña et al. (2000) tenha sido menor, essa medida para PES só foi alcançada quando os animais atingiram $635 \mathrm{~kg}$ de PS, ajustado para 570 dias de idade.
Verifica-se que aos 590 dias de idade, o PES encontra-se em fase adiantada de desenvolvimento, tendendo à estabilização. Segundo Coulter (1986), o perímetro escrotal estabiliza-se em pesos menores nas raças mais precoces, quando comparadas às mais tardias. Dessa forma, e de acordo com Dal-Farra et al. (1998), sua mensuração mais cedo, dos 520 aos 550 dias de idade, por exemplo, poderia otimizar a identificação dos animais mais precoces.

Os fatores de correção para PES, de acordo com GG, HI, PS e IDS, são apresentados na Tab. 3 . Observa-se que a magnitude dos fatores de correção estimados variou de 0,0511 para HI a 0,2353, para PS. Amplitudes semelhantes foram observadas por Dal-Farra et al. (1998) para animais da raça Nelore. Ortiz-Peña et al. (2000) 
verificaram amplitude de 1,22, para peso aos 570 dias de idade, apresentando maior heterogeneidade no peso corporal, havendo maior variação deste em menor intervalo de idade.

A utilização dos fatores de correção do perímetro escrotal é apresentada na Tab. 4. Verifica-se que entre os indivíduos com mesmo PES e mesma IDS, após a correção para peso corporal, o de menor peso ficou melhor classificado. Por outro lado, para animais com perímetros e pesos iguais e idades diferentes, a correção privilegiou o animal mais novo. Deve-se ressaltar que, segundo Ortiz-Peña et al. (2000), a correção do PES apenas para idade pode levar ao aumento do tamanho adulto das vacas, o que eleva suas exigências de mantença e, consequentemente, os custos da criação. Por isso, a utilização do PES ajustado para peso e idade seria mais adequada que a correção deste apenas para peso ou idade, permitindo o aumento da precocidade sexual sem elevar o peso e o tamanho adulto dos animais.

Tabela 3. Fatores de correção (FC) do perímetro escrotal para grupo genético (GG), heterozigose individual (HI), peso ao sobreano em quilogramas (PS) e idade à pesagem de sobreano em dias (IDS)

\begin{tabular}{cccccccc}
\hline GG & FC & HI & FC & PS & FC & IDS & FC \\
\hline 0,0000 & 1,0000 & 0,0000 & 1,0000 & 240 & 1,1174 & 340 & 1,1642 \\
0,1875 & 1,0291 & 0,2500 & 0,9867 & 280 & 1,0583 & 380 & 1,1289 \\
0,2500 & 1,0392 & 0,3750 & 0,9802 & 320 & 1,0105 & 420 & 1,0957 \\
0,3125 & 1,0495 & 0,5000 & 0,9738 & 360 & 0,9718 & 460 & 1,0643 \\
0,3750 & 1,0599 & 0,6250 & 0,9674 & 400 & 0,9406 & 500 & 1,0347 \\
0,4375 & 1,0707 & 0,7500 & 0,9612 & 440 & 0,9158 & 540 & 1,0068 \\
0,5000 & 1,0816 & 0,8750 & 0,9550 & 480 & 0,8965 & 580 & 0,9802 \\
0,6250 & 1,1042 & 1,0000 & 0,9489 & 520 & 0,8821 & 620 & 0,9551 \\
0,6875 & 1,1158 & - & - & 560 & 0,8721 & 660 & 0,9312 \\
0,7500 & 1,1276 & - & - & 600 & 0,8664 & 700 & 0,9085 \\
- & - & - & - & 640 & 0,8646 & 740 & 0,8868 \\
\hline
\end{tabular}

Tabela 4. Utilização dos fatores de correção do perímetro escrotal ao sobreano (PES) para grupo genético (GG), heterozigose individual (HI), peso ao sobreano em quilogramas (PS) e idade à pesagem de sobreano em dias (IDS)

\begin{tabular}{ccccccc}
\hline Animal & $\begin{array}{c}\text { PES observado } \\
(\mathrm{cm})\end{array}$ & GG & HI & PS & IDS & $\begin{array}{c}\text { PES observado } \\
(\mathrm{cm})\end{array}$ \\
\hline A & 28 & 0,0000 & 0,0000 & 330 & 550 & 28,00 \\
B & 28 & 0,7500 & 0,0000 & 330 & 550 & 31,57 \\
C & 28 & 0,5000 & 0,0000 & 330 & 550 & 30,28 \\
D & 28 & 0,5000 & 1,0000 & 330 & 550 & 28,74 \\
E & 28 & 0,0000 & 0,0000 & 360 & 420 & 29,81 \\
F & 28 & 0,0000 & 0,0000 & 360 & 380 & 30,72 \\
G & 28 & 0,0000 & 0,0000 & 360 & 460 & 28,96 \\
H & 28 & 0,0000 & 0,0000 & 440 & 460 & 27,29 \\
\hline
\end{tabular}

\section{CONCLUSÕES}

Os resultados evidenciam a necessidade de corrigir as medidas de perímetro escrotal para os efeitos de: grupo genético, heterozigose individual, peso corporal e idade ao sobreano; para que essa característica possa ser utilizada como critério de seleção para precocidade sexual.

\section{AGRADECIMENTOS}

Os autores agradecem à Gensys Consultores Associados S/C Ltda. e Natura Genética SulAmericana pela cessão do Banco de Dados e à CAPES e ao CNPq pela concessão de bolsas de Mestrado e de Iniciação Científica, respectivamente. 


\section{REFERÊNCIAS BIBLIOGRÁFICAS}

ALENCAR, M.M.; BARBOSA, P.F.; BARBOSA, R.T. et al. Parâmetros genéticos para peso e circunferência escrotal em touros da raça Canchim. Rev. Bras. Zootec., v.22, p.572$583,1993$.

BERGMANN, J.A.G. Indicadores de precocidade sexual em bovinos de corte. In: CONGRESSO BRASILEIRO DE RAÇAS ZEBUÍNAS, 3., 1999, Uberaba. Anais... Uberaba, 1999. p.145-155.

BRITO, F.V. Influência da idade e peso corporal sobre o perímetro escrotal em touros Hereford estimativas de fatores de correção. In: REUNIÃO ANUAL DA SOCIEDADE BRASILEIRA DE ZOOTECNIA, 34., 1997, Juiz de Fora, MG. Anais... Juiz de Fora, SBZ, 1997. p.130-132,

COULTER, G.H. Puberty and postpuberal development of beef bulls. In: MORROW, D.A. (Ed.). Current therapy of theriogenology. 2.ed. Philadelphia: W.B. Saunders, 1986. p.142-148.

COULTER, G.H.; KELLER, D.G. Scrotal circumference of young beef bulls; relationship to paired testes weight effect to breed, and predictability. Can. J. Anim. Sci., v.62, p.133139, 1982.

CUNDIFF, L.C.; GREGORY, K.E.; SCHWULST, F.J. et al. Effects of heterosis on maternal performance and milk production in Hereford, Angus and Shorthorn Cattle. J. Anim. Sci., v.38, p.729-745, 1974.

DAL-FARRA, R.A.; FRIES, L.A; LOBATO, J.F.P. Fatores de correção do perímetro escrotal para efeitos de idade e peso ao sobreano de tourinhos Nelore. Rev. Bras. Zootec., v.27, p.1092-1096, 1998.

DAL-FARRA, R.A.; ROSO, V.M.; KILPP, D.V. Fatores de correção do perímetro escrotal para efeitos de idade, peso e heterozigose individual, em touros mestiços Angus x Nelore. Rev. Bras. Zootec., v.29, supl.1, p.2002-2007, 2000.
DICKERSON, G.E. Inbreeding and heterosis in animals. In: ANIMAL BREEDING AND GENETICS SYMPOSIUM IN HONOR OF DR. J.L. LUSH, 1973, Champaign, IL. Proceedings... Champaign, IL: American Society of Animal Science, 1973. p.54-77.

GREGORY, K.E.; CUNDIFF, L.V.; KOCH, R.M. Composite breed to use heterosis and breed diferences to improve efficiency of beef production. Nebraska: Agricultural Research Service and University of Nebraska, 1995. 80p.

LUNSTRA, D.D.; GREGORY, K.E.; CUNDIFF, L.V. Heritability estimates and adjustments factors for the effects of bull age and age of dam on yearling testicular size in breeds of bulls. Theriogenology, v.30, p.127-136, 1988.

ORTIZ-PEÑA, C.D.; QUEIROZ, S.A.; FRIES, L.A. Estimação de fatores de correção do perímetro escrotal para idade e peso corporal em touros jovens da raça nelore. Rev. Bras. Zootec., v.29, p.1667-1675, 2000.

PEREIRA, E.; ELER, J.P.; COSTA, F.A.A. et al. Análise genética da idade ao primeiro parto e do perímetro escrotal em bovinos da raça Nelore. Arq. Bras. Med. Vet. Zootec., v.53, p.116-121, 2001.

SKRYPZECK, H.; SCHOEMAN, S.J.; JORDAAN, G.F. et al. Estimates of crossbreeding parameters in a multibreed beef cattle crossbreeding project. S. Afr. J. Anim. Sci., v.30, p.193-203, 2000.

TEIXEIRA, R.A.; DIAS, L.; ALBUQUERQUE, L.G. Efeitos do peso e idade à desmama e ao sobreano, sobre medida de perímetro escrotal em touros Nelore. In: REUNIÃO ANUAL DA SOCIEDADE BRASILEIRA DE ZOOTECNIA, 35., 1998, Botucatu, SP. Anais... Botucatu: SBZ, 1998. p.443-445.

TOELLE, V.D.; ROBINSON, O.W. Estimates of genetic correlation between measurements and female reproductive traits in cattle. J. Anim. Sci., v.60, p.89-100, 1985. 